\title{
Ion Radical Cleavage Reactions. III. The Cleavage of Halide Ion from Mono- and Dihalobenzophenone Anion Radicals
}

\author{
BJØRG AALSTAD and VERNON D. PARKER
}

Laboratory for Organic Chemistry, Norwegian Institute of Technology, University of Trondheim, N-7034 Trondheim-NTH, Norway

Benzophenone anion radicals substituted in the 4or $4,4^{\prime}$-positions with either chlorine or bromine undergo cleavage in acetonitrile or $N, N$ dimethylformamide to generate the corresponding aryl radicals. Cleavage of the monochlorosubstituted anion radical is $6-7$ times faster than that of the corresponding disubstituted anion radical and bromo-substituted anion radicals cleave about $10^{3}$ times faster than the corresponding chloro derivatives. A very small solvent effect was found with the reactions being somewhat slower in acetonitrile. Arrhenius activation energies are very similar for mono- and disubstituted anion radicals but about $8 \mathrm{kcal} / \mathrm{mol}$ greater for chloro substitution than for the corresponding bromo-substituted anion radicals. Entropies of activation were observed to be close to zero for the chlorosubstituted anion radicals but of the order of $-(12$ $-20) \mathrm{cal} / \mathrm{K}$ mol for the bromo derivatives. The more negative values of $\Delta S_{298}^{*}$ in the latter case were interpreted to indicate that bond cleavage is much further advanced in the transition state in the case of bromo-substituted anion radicals.

In other papers in this series, the cleavage reactions of halo-substituted anthracene ${ }^{1}$ and nitrobenzene ${ }^{2}$ anion radicals have been examined. The two reaction series were observed to be similar in that the bromo derivatives were in both cases $>10^{3}$ times as reactive as the corresponding chlorosubstituted anion radicals. However, the two differed sharply in the role of the entropy of activation in determining the reaction rates. In the case of the nitrobenzene anion radicals, $\Delta S_{298}^{\neq}$was small and positive regardless of the nature or the location of the halogen substituent. In contrast $\Delta S_{298}^{*}$ for the cleavage of 9-bromo- or 9,10 dibromoanthracene anion radicals was of the order of $21 \mathrm{cal} / \mathrm{K}$ mol more negative than for the corresponding chloro-substituted anion radicals. The large negative entropies of activation for the bromoanthracene anion radicals was taken to indicate a high degree of bond cleavage in the transition state. Thus, the position of the transition state in that series of reactants is very much dependent upon the nature of the halogen substituent. On the other hand, the near independence of $\Delta S_{298}^{*}$ for the reactions of nitrobenzene anion radicals on the substituent must indicate that the position of the transition state along the reaction coordinate does not vary significantly with the nature of the substituent. The differences in the two reaction series can then be a reflection of the differences in charge distributions in the anion radicals. The nitrobenzene anion radicals have highly polarized structures with most of the charge concentrated on the heteroatoms while the anthracene anion radicals apparently have a more dispersed charge distribution. However, it is still not clear why the structures of the transition states for the cleavage reactions in the latter series are so strongly dependent upon the leaving group.

The 4- and 4,4'-substituted benzophenones, structures 1 and 2, would be expected to form anion radicals with charge distributions intermediate between those of the highly polarized nitrobenzenes and the more dispersed charge encountered in the anthracenes. Another interesting feature of the structures of these anion radicals as compared to the

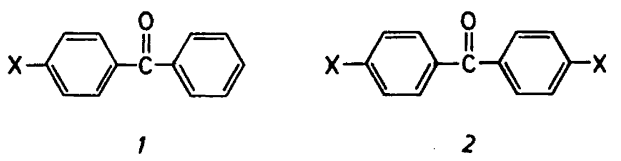


anthracenes is that in this case the disubstituted anion radicals have substituents in two different rings which are not in complete conjugation because of interactions between the 2- and 2'-hydrogen atoms which cause the two rings to lie in different planes.

The cleavage reactions of mono-halobenzophenones anion radicals have been studied intensively by Savéant and co-workers. ${ }^{3-5}$ The reaction pathway has been convincingly demonstrated to be a simple unimolecular cleavage reaction to yield the reactive aryl radical as in eqn. (1). The aryl radical can then undergo further electron transfer (2) or abstract hydrogen atoms from the solvent (3). The conditions under which the two competitive reactions (2) and (3) operate have been analyzed. ${ }^{6}$ Since the cleavage reaction (1) is rate-determining, the kinetics and activation parameters are expected to be a direct reflection of this microscopic step as in the case of the haloanthracene anion radicals.

$$
\begin{aligned}
& 1 \\
& \mathrm{Ar}-\mathrm{X}^{-}-\stackrel{k_{1}}{\rightarrow} \mathrm{Ar}+\mathrm{X}^{-} \\
& \mathrm{Ar}^{-}+\mathrm{ArX}^{-} \rightarrow \mathrm{Ar}^{-}+\mathrm{ArX} \\
& \mathrm{Ar}^{-}+\mathrm{S}-\mathrm{H} \rightarrow \mathrm{ArH}+\mathrm{S}
\end{aligned}
$$

With regard to our interest in these reactions, i.e. the activation parameters and the nature of the transition states, some very interesting observations were made in the previous studies. The reactions were reported to be from 30 to 80 times faster in $\mathrm{N}, \mathrm{N}$-dimethylformamide (DMF) than in acetonitrile (AN). ${ }^{3}$ Although activation parameters were not determined, the rate constants at -10 and $+20^{\circ} \mathrm{C}$ correspond to an energy of activation for both 3-bromo- and 4-bromobenzophenone anion radicals in DMF equal to about $21 \mathrm{kcal} / \mathrm{mol}$, an unusually high value for the rapid reactions and requires $\Delta S_{298}^{*}$ of from 20 to $31 \mathrm{cal} / \mathrm{K} \mathrm{mol}$. On the other hand, data for measurements in liquid ammonia $^{5}$ for the cleavage of 4-bromobenzophenone anion radical indicate that $E_{\mathrm{a}}$ in that solvent is about $13 \mathrm{kcal} / \mathrm{mol}$ and $\Delta S_{298}^{\neq}$is $2 \mathrm{cal} / \mathrm{K}$ mol. It should be pointed out that the rate constants of previous studies ${ }^{3,5}$ were measured by ordinary cyclic voltammetry and large error limits were given. This, of course, is a good reason for not reporting activation parameters and the values quoted here must be considered with that in mind. However, the data did serve to stimulate our curiousity and a reinvestigation appeared necessary.

We chose to investigate mono- and disubstituted anion radicals of 1 and 2 for two reasons. As in the case of the anthracene anion radical cleavage reactions, we anticipated that the dibromo derivative would be less reactive than 4bromobenzophenone anion radical and thus make the rate data more reliable. Also, the effect of the second halo substituent on the activation parameters was expected to shed some light on the nature of the transition states for the cleavage reactions. There have been no previous reports of data for the cleavage reactions of the anion radicals of 2 .

\section{EXPERIMENTAL}

The monohalobenzophenones were reagent grade and were recrystallized before use. The dihalobenzophenones were generously provided by Dr. Torkil Holm.

Solvents containing electrolyte, $\mathrm{Bu}_{4} \mathrm{NBF}_{4}(0.1 \mathrm{M})$, were passed through a column of neutral alumina (Woelm W 200, neutral, super grade 1) before adding the substrate and carrying out kinetic studies.

The temperature was controlled either by a Haake cryostat $\left(<0^{\circ} \mathrm{C}\right)$ or by suitable water baths maintained at constant temperature over the relatively short measurement times by virtue of the large heat capacity of the baths.

The cells, reference electrodes, working electrodes, and the data retrieval system have been described in recent papers from this laboratory. ${ }^{7,8}$

\section{RESULTS}

Kinetic Measurements. The kinetics of the cleavage reactions were studied by derivative cyclic voltammetry $^{9}$ using procedures recently described. ${ }^{10}$ Difficulty was encountered in evaluating rate constants for the bromo-substituted anion radicals because the oxidation peak due to the anion radical of the dehalogenated product overlapped with that for the primary intermediate. This evidently was also the source of much of the error in rate constants previously reported for 4bromobenzophenone anion radical cleavage. We found that the problem could be minimized by evaluating rate constants after little reaction had 
taken place. The procedure used was to determine $v_{3 / 4}$, i.e. the voltage sweep rate necessary for the derivative peak ratio, $R_{\mathrm{l}}^{\prime}$, to equal 0.750 . Rate constants could then be evaluated from eqn. (4)

$k=515.6 v_{3 / 4} / T$

determined from theoretical data. Rate constants evaluated from $v_{3 / 4}$ were found to be within experimental error of those calculated from eqn. (5)

$k=1444 v_{1 / 2} / T$

using $v_{1 / 2}$, as is usually done, ${ }^{10}$ when the substrate was 4,4'-dichlorobenzophenone. A disadvantage of this procedure for rapid reactions is that the voltage sweep rate required to evaluate the rate constant is almost 3 times greater when (4) is used than when $v_{1 / 2}$ is determined. On the other hand, the data are very much more reliable when the effect of the interfering reactions is minimized.

Error analysis. In general, the values of $R_{1}^{\prime}$ used in the evaluation of rate constants were based on sufficient data so that the mean values were 0.750 \pm 0.002 which corresponds to about a $1 \%$ error in $k$. Thus the error in the measurement of $R_{\mathrm{I}}^{\prime}$ is nearly negligible. In order to get an estimate of the error from all sources we can consider the data in Table 1. The maximum deviation of rate constants determined during the reduction of 4,4'dichlorobenzophenone for duplicate runs was observed to be about $2 \%$ at $0.50 \mathrm{mM}$ and less than $1 \%$ at the higher concentrations. However, rate constants evaluated at a substrate concentration of $0.50 \mathrm{mM}$ were about $10 \%$ lower than those

Table 1. Reaction order analysis of the cleavage of 4,4'-dichlorobenzophenone anion radical in $\mathrm{DMF}^{a}$

\begin{tabular}{lll}
\hline$C_{\mathrm{A}} / \mathrm{mM}$ & $v_{\frac{1}{2}} / \mathrm{V} \mathrm{s}^{-1}$ & $k / \mathrm{s}^{-1}$ \\
\hline 0.50 & 1.107 & 5.45 \\
0.50 & 1.078 & 5.30 \\
1.00 & 1.128 & 5.55 \\
1.00 & 1.138 & 5.60 \\
2.00 & 1.232 & 6.06 \\
2.00 & 1.231 & 6.06 \\
\hline
\end{tabular}

${ }^{a}$ Measurements by derivative cyclic voltammetry in solvent containing $\mathrm{Bu}_{4} \mathrm{NBF}_{4}(0.1 \mathrm{M})$ at a mercury electrode at $22^{\circ} \mathrm{C}$. The duplicate runs at each substrate concentration were on separately prepared solutions. The difference in switching and reversible potentials, $E_{\mathrm{sw}}-E_{\mathrm{rev}}$ was $200 \mathrm{mV}$ in all cases. obtained at $2.00 \mathrm{mM}$. The reason for this deviation does not appear to be measurement precision but rather some other complicating factors. The measurements involving the chloro-substituted derivatives are more reliable than those on the bromo derivatives because of the lower temperatures and higher sweep rates required for the latter. All comparisons of rate constants were made at the same substrate concentration, $2.00 \mathrm{mM}$. Under these conditions $k$ is believed to be reproducible to $\pm 1 \%$ for the chloro-substituted anion radicals and about $\pm 10 \%$ for the corresponding bromo derivatives.

Reaction order analysis. The criterion for a first order reaction of the anion radical generated during cyclic voltammetric reduction of the substrate is that the voltage sweep rate necessary for a particular value of the derivative peak ratio should be independent of the substrate concentration. ${ }^{10}$ The data in Table 1 are for the reduction of 4,4'dichlorobenzophenone in DMF for the case where $v_{1 / 2}$ was measured as a function of substrate concentration. The value of $v_{1 / 2}$ increased by about $12 \%$ in going from $0.50 \mathrm{mM}$ to $2.00 \mathrm{mM}$. As pointed out in the previous paragraph, the variation observed is clearly larger than experimental error. The data suggest that either there is a minor competing second order reaction or that oxidation of products is beginning to interfere at the higher concentrations. In any case the complication is not very severe.

Table 2. Kinetic data for the cleavage of halobenzophenone anion radicals in DMF. ${ }^{a}$

\begin{tabular}{llcc}
\hline Substituents & $T / \mathrm{K}$ & $v_{\frac{1}{2}}^{b} / \mathrm{V} \mathrm{s}^{-1}$ & $\mathrm{k} / \mathrm{s}^{-1}$ \\
\hline 4-Chloro & 296.2 & 8.67 & 42.3 \\
4-Chloro & 304.4 & 19.0 & 90.1 \\
4-Chloro & 314.2 & 42.2 & 194 \\
4,4'-Dichloro & 295.5 & 1.20 & 5.84 \\
4,4'-Dichloro & 304.4 & 2.80 & 13.3 \\
4,4'-Dichloro & 312.4 & 5.71 & 26.4 \\
4,4'-Dichloro & 319.6 & 8.70 & 39.3 \\
4,4'-Dibromo & 234.8 & $220^{c}$ & 483 \\
4,4'-Dibromo & 229.9 & $160^{\mathrm{c}}$ & 359 \\
4,4'-Dibromo & 224.9 & $110^{\mathrm{c}}$ & 252 \\
\hline
\end{tabular}

${ }^{a}$ Conditions as in Table $1 .^{b}$ The voltage sweep rate necessary for $R_{\mathrm{I}}^{\prime}=0.500 .^{c}$ The voltage sweep rate necessary for $R_{\mathrm{I}}^{\prime}=0.750, v_{3 / 4}$, was used in these analyses due to the interference of further reaction products at higher conversions. 
Table 3. Kinetic data for the cleavage of halobenzophenone anion radicals in acetonitrile. ${ }^{a}$

\begin{tabular}{lccc}
\hline Substituents & $T / \mathrm{K}$ & $v_{3 / 4}{ }^{b} / \mathrm{V} \mathrm{s}^{-1}$ & $k / \mathrm{s}^{-1}$ \\
\hline 4-Chloro & 294.9 & 14.2 & 24.8 \\
4-Chloro & 304.0 & 31.0 & 52.5 \\
4-Chloro & 311.0 & 69.7 & 115.5 \\
4-Chloro & 321.9 & 151.9 & 243.3 \\
4,4'-Dichloro & 293.5 & 2.20 & 3.87 \\
4,4'-Dichloro & 304.9 & 6.32 & 10.7 \\
4,4'-Dichloro & 313.7 & 14.0 & 23.0 \\
4,4'-Dichloro & 320.3 & 25.6 & 41.2 \\
4-Bromo & 225.0 & 109 & 250 \\
4-Bromo & 229.3 & 180 & 405 \\
4-Bromo & 233.3 & 235 & 520 \\
4-Bromo & 243.8 & 490 & 1037 \\
4,4'-Dibromo & 233.5 & 72.0 & 159 \\
4,4'-Dibromo & 238.4 & 109 & 236 \\
4,4'-Dibromo & 243.4 & 163 & 346 \\
\hline
\end{tabular}

${ }^{a}$ Conditions as in Table 1. ${ }^{b}$ Defined in Table 2.

Rate constants for the cleavage reactions. Kinetic data for the cleavage reactions in DMF and AN are summarized in Tables 2 and 3, respectively. Rate data are not reported for 4-bromobenzophenone anion radical in DMF since $v_{3 / 4}$ of the order of $500 \mathrm{~V}$ $\mathrm{s}^{-1}$ or more were necessary at the low temperatures and the data did not appear to be of sufficiently high quality for the determination' of activation parameters. In DMF, $v_{1 / 2}$ was evaluated for the chloro-substituted anion radicals and $v_{3 / 4}$ was used for the analysis of the data for the cleavage of 4,4'dibromobenzophenone anion radical. In $\mathrm{AN}, v_{3 / 4}$ was the basis for all of the rate constant determinations.

Activation parameters for the cleavage reactions The energy of activation for the cleavage of the chloro- substituted anion radicals was very nearly the same in all cases, independent of the solvent. The mean of the four values, $16.2 \pm 0.4$, suggests that the value for the 4-chloro derivative in DMF is on the low side. If the mean $E_{\mathrm{a}}$ is used in the calculation of $\Delta S_{298}^{\neq}$in that case the value goes from -0.4 to $+1.7 \mathrm{cal} / \mathrm{K}$ mol. However, this is probably extending the expected precision too far. The error in $E_{\mathrm{a}}$ is estimated to be of the order of $\pm 0.5 \mathrm{kcal} / \mathrm{mol}$ and that for $\Delta S_{298}^{*}$ is then of the order of $\pm 1 \mathrm{cal} / \mathrm{K} \mathrm{mol}$ for the reactions involving cleavage of chloride ion. Thus, $\Delta S_{298}^{\neq}$appears to be from 1 to $3 \mathrm{cal} / \mathrm{K} \mathrm{mol}$ more negative for the dichloro-substituted anion radical than for the monochloro derivative.

On the other hand, the data for the cleavage reactions of the bromo-substituted anion radicals are clearly of lower quality. In this case $E_{\mathrm{a}}$ was observed to be equal to $8.0 \pm 1.0 \mathrm{kcal} / \mathrm{mol}$. If this value is used in the calculation of the entropy of activation in all three cases, values of -13.8 for 4 bromobenzophenone anion radical and -15.2 or $-15.1 \mathrm{cal} / \mathrm{K} \mathrm{mol}$ are found for 4,4'-dibromobenzophenone anion radical. This suggests that the $E_{\mathrm{a}}$ values measured in this case are within experimental error of being the same and that the $\Delta S_{298}^{\neq}$is more negative for bromo-substituted 2 than for 1 anion radicals. Furthermore, it appears that the cleavage of bromide ion is accompanied by an entropy of activation of the order of $14 \mathrm{cal} / \mathrm{K} \mathrm{mol}$ more negative than the corresponding cleavage of chloride ion.

\section{DISCUSSION}

Contrary to expectations based on previous work, ${ }^{3,5}$ we find a very small solvent effect on the cleavage reactions. An increase of less than $50 \%$ in

Table 4. Activation parameters for the cleavage of halo-benzophenone anion radicals. ${ }^{a}$

\begin{tabular}{lllccc}
\hline Substituents & Solvent & $E_{\mathrm{a} / \mathrm{kcal} / \mathrm{mol}}$ & $k_{298} / \mathrm{s}^{-1}$ & $\Delta S_{298}^{\neq}{ }^{b}$ & $\begin{array}{l}\text { Correlation } \\
\text { coefficient }\end{array}$ \\
\hline 4-Chloro & AN & 16.3 & 33.5 & 1.0 & -0.9968 \\
4-Chloro & DMF & 15.6 & 51.2 & -0.4 & -0.9996 \\
4,4'-Dichloro & AN & 16.4 & 5.94 & -2.0 & -0.9998 \\
4,4'-Dichloro & DMF & 16.3 & 7.53 & -1.7 & -1.0000 \\
4-Bromo & AN & 8.0 & 21700 & -13.8 & -0.9942 \\
4,4'-Dibromo & AN & 9.0 & 10900 & -11.7 & -1.0000 \\
4,4'-Dibromo & DMF & 6.9 & 11300 & -18.9 & -0.9995 \\
\hline
\end{tabular}

${ }^{a}$ Arrhenius correlation of data from Tables 2 and $3 .{ }^{b} \mathrm{In} \mathrm{cal} / \mathrm{K} \mathrm{mol}$. 
the rate constants for the cleavage of chloride ion was observed in going from DMF to AN. In fact, if we compare the rate constant we obtain at $-40^{\circ} \mathrm{C}$ for the cleavage of bromide from 4-bromobenzophenone anion radical in AN with that reported for the same reaction in liquid ammonia, ${ }^{5}$ we find that the values, 520 and $590 \mathrm{~s}^{-1}$ are surely within experimental error of being the same. We conclude that the large differences observed earlier were most likely due to the fact that the products of the reaction interfere severely with analysis. The interference is especially severe when peak current ratios are evaluated after considerable reaction has occurred. This is normally the way cyclic voltammetric data are evaluated and in this case the analysis must be very uncertain.

The activation energies that we find for the cleavage of bromide ion from benzophenone anion radicals, $8.0 \pm 1.0 \mathrm{kcal} / \mathrm{mol}$, are also much lower than those predicted from previous work in any of the solvents. This again, can be attributed to precision problems.

We do find that the activation parameters for the cleavage of halobenzophenone anion radicals are intermediate between those found for the anthracene series and the nitrobenzene series. However, the trends in the data resemble those found for the haloanthracene anion radicals. For the anthracene series, $E_{\mathrm{a}}$ was about $11 \mathrm{kcal} / \mathrm{mol}$ lower for the cleavage of bromide ion, $\Delta S_{298}^{\neq}$was about $21 \mathrm{cal} / \mathrm{K}$ mol more negative and cleavage from dihalo derivatives was accompanied by about $4 \mathrm{cal} / \mathrm{K} \mathrm{mol}$ more negative $\Delta S_{298}^{*}$. The corresponding differences appear to be $8 \mathrm{kcal} / \mathrm{mol},-14 \mathrm{cal} / \mathrm{K} \mathrm{mol}$ and -2 $\mathrm{cal} / \mathrm{K} \mathrm{mol}$, respectively, for the cleavage of halide ion from benzophenone anion radicals.

Because of the very close similarity in the reactions considered here to those of the haloantracene anion radicals, ${ }^{1}$ the data reported serve to reinforce the conclusions drawn from the previous study. The standard entropy of formation of ion radicals of aromatic compounds has been observed to be small in acetonitrile, usually less than $10 \mathrm{cal} / \mathrm{K} \mathrm{mol}$, and negative in the case of anion radicals. ${ }^{11}$ Values of -46.7 and $-43.3 \mathrm{cal} / \mathrm{K} \mathrm{mol}$ have been given for chloride and bromide, respectively, in DMF. ${ }^{12}$ Thus, the entropy of reaction for the cleavage of halide ion from a haloaromatic anion radical would be expected to be of the order of $-40 \mathrm{cal} / \mathrm{K} \mathrm{mol}$ in DMF and similar in AN. If cleavage of the carbon-halogen bond is essentially complete in the transition state the entropy of activation is expected to be large and negative approaching about $-40 \mathrm{cal} / \mathrm{K} \mathrm{mol}$ in the limit. On the other hand, the observation of a positive or small entropy of activation implies that bond cleavage is not very far advanced in the transition state. Thus, the entropy of activation serves as a good measure of the position of the transition state along the reaction coordinate in these reactions.

As in the case of the cleavage of haloanthracene anion radicals, a nearly zero entropy of activation is observed for the cleavage of the chlorobenzophenone anion radicals in both DMF and AN. This implies that there is not very much reorganization of the solvent in the transition state as compared to the anion radical. The logical interpretation then is that an early transition state is involved in this case. On the other hand, activation entropies ranging from -12 to -19 were found for the corresponding reactions where bromide is the leaving group. The larger negative entropies of activation in this case imply that there is considerable solvent reorganization upon going to the transition state. The latter means that bond cleavage is rather far advanced.

We conclude that halobenzophenone anion radicals resemble those of the haloanthracenes quite closely with respect to cleavage reactions. The reason for the apparently large differences in the structures of the transition states for chloride and bromide cleavage is still not apparent. It is hoped that further work in related systems will shed light on this perplexing question.

\section{REFERENCES}

1. Parker, V. D. Acta Chem. Scand. B 35 (1981) 595.

2. Parker, V. D. Acta Chem. Scand. B 35 (1981) 655.

3. Nadjo, L. and Savéant, J. M. J. Electroanal. Chem. 30 (1971) 41.

4. M'Halla, F., Pinson, J. and Savéant, J. M. J. Electroanal. Chem. 89 (1978) 347.

5. Savéant, J. M. and Thiébault, A. J. Electroanal. Chem. 89 (1978) 335.

6. M'Halla, F., Pinson, J. and Savéant, J. M. J. Am. Chem. Soc. 102 (1980) 4120.

7. Aalstad, B., Ronlán, A. and Parker, V. D. Acta Chem. Scand. B 35 (1981) 247.

8. Ahlberg, E. and Parker, V. D. Acta Chem. Scand. B 34 (1980) 97.

9. Ahlberg, E. and Parker, V. D. J. Electroanal. Chem. 121 (1981) 57. 
10. Parker, V. D. Acta Chem. Scand. B 35 (1981) 233.

11. Svaan, M. and Parker, V. D. Acta Chem. Scand. B 35 (1981) 559.

12. Criss, C. M. In Covington, A. K. and Dickinson, T., Eds., Physical Chemistry of Organic Solvent Systems, Plenum, London 1973, Chapter 2, Table 2.12.1.

Received October 7, 1981. 